Research

\title{
Changes in pulse pressure variability during cardiac resynchronization therapy in mechanically ventilated patients
}

\author{
Cornelius Keyl1, Jochem Stockinger², Sven Laule ${ }^{1}$, Klaus Staier ${ }^{1}$, Jochen Schiebeling-Römer ${ }^{2}$ and \\ Christoph Wiesenack ${ }^{3}$
}

\author{
1Department of Anesthesiology, Heart Centre Bad Krozingen, Suedring 15, 79189 Bad Krozingen, Germany \\ 2Department of Rhythmology, Heart Centre Bad Krozingen, Suedring 15, 79189 Bad Krozingen, Germany \\ ${ }^{3}$ Department of Anesthesiology, University Hospital Regensburg, Franz-Josef-Strauss-Allee 11, 93042 Regensburg, Germany \\ Corresponding author: Cornelius Keyl, cornelius.keyl@herzzentrum.de
}

Received: 13 Jan 2007 Revisions requested: 27 Feb 2007 Revisions received: 20 Mar 2007 Accepted: 19 Apr 2007 Published: 19 Apr 2007

Critical Care 2007, 11:R46 (doi:10.1186/cc5779)

This article is online at: http://ccforum.com/content/11/2/R46

(C) 2007 Keyl et al.; licensee BioMed Central Ltd.

This is an open access article distributed under the terms of the Creative Commons Attribution License (http://creativecommons.org/licenses/by/2.0), which permits unrestricted use, distribution, and reproduction in any medium, provided the original work is properly cited.

\begin{abstract}
Introduction The respiratory variation in pulse pressure (PP) has been established as a dynamic variable of cardiac preload which indicates fluid responsiveness in mechanically ventilated patients. The impact of acute changes in cardiac performance on respiratory fluctuations in PP has not been evaluated until now. We used cardiac resynchronization therapy as a model to assess the acute effects of changes in left ventricular performance on respiratory PP variability without the need of pharmacological intervention.

Methods In 19 patients undergoing the implantation of a biventricular pacing/defibrillator device under general anesthesia, dynamic blood pressure regulation was assessed during right ventricular and biventricular pacing in the frequency domain (power spectral analysis) and in the time domain (PP variation: difference between the maximal and minimal PP values, normalized by the mean value).
\end{abstract}

Results PP increased slightly during biventricular pacing but without statistical significance (right ventricular pacing, $33 \pm 10$ $\mathrm{mm} \mathrm{Hg}$; biventricular pacing, $35 \pm 11 \mathrm{~mm} \mathrm{Hg}$ ). Respiratory PP fluctuations increased significantly (logarithmically transformed $P P$ variability $-1.27 \pm 1.74 \mathrm{ln} \mathrm{mm} \mathrm{Hg}^{2}$ versus $-0.66 \pm 1.48 \mathrm{ln} \mathrm{mm}$ $\left.\mathrm{Hg}^{2} ; p<0.01\right)$; the geometric mean of respiratory PP variability increased 1.8-fold during cardiac resynchronization. PP variation, assessed in the time domain and expressed as a percentage, showed comparable changes, increasing from $5.3 \%(3.1 \% ; 12.3 \%)$ during right ventricular pacing to $6.9 \%$ $(4.7 \% ; 16.4 \%)$ during biventricular pacing (median [25th percentile; 75th percentile]; $p<0.01$ ).

Conclusion Changes in cardiac performance have a significant impact on respiratory hemodynamic fluctuations in ventilated patients. This influence should be taken into consideration when interpreting PP variation.

\section{Introduction}

The respiratory fluctuations of stroke volume and its surrogate, pulse pressure (PP), in mechanically ventilated patients are an expression of the relationship between changes in left ventricular preload and stroke volume. Several studies have found a significant correlation between PP variation or stroke volume variation and the increase in cardiac output caused by fluid loading [1-3]. Therefore, respiratory fluctuations of cardiovascular parameters are accepted measures of cardiac volume responsiveness in mechanically ventilated patients [4].

However, preload is only one determinant of cardiac performance (besides ventricular contractility and afterload proper- ties). Whereas the influence of changes in preload on the variation of stroke volume, $\mathrm{PP}$, or systolic blood pressure (SBP) has been investigated in detail, the extent to which these dynamic measures are modified by changes in ventricular performance is not yet clear. This might be due to the fact that the treatment with inotropic drugs also modifies heart rate and vascular tone, thus making it impossible to study the isolated effect of changes in cardiac performance on dynamic cardiovascular measures.

We used cardiac resynchronization therapy as a model to study the effect of changes in cardiac performance on the variation of hemodynamic variables. Cardiac resynchronization therapy is an accepted therapeutic approach for improving

$\overline{\mathrm{ECG}}=$ electrocardiogram; $\mathrm{PP}=$ pulse pressure; $\mathrm{SBP}=$ systolic blood pressure. 
cardiac performance in patients with heart failure associated with an intraventricular conduction disorder [5-7]. In the present study, we compared the acute effects of right ventricular and biventricular pacing on PP fluctuations in mechanically ventilated patients with impaired myocardial function. We were thus able to assess the influence of changes in cardiac contractility on static and dynamic cardiocirculatory parameters without changing heart rate, vascular tone, or intravascular volume status.

\section{Materials and methods}

After obtaining the approval of the local ethics committee and written informed consent, we studied 19 patients (15 men, ages 51 to 78 years) with New York Heart Association class III (18 patients) and class IV (1 patient) heart failure and dyssynchrony between right and left ventricular contractions who were scheduled for the implantation of a combined biventricular pacing/defibrillator device (Contak Renewal; Guidant $\mathrm{GmbH}$, Giessen, Germany).

The patients underwent routine monitoring by means of electrocardiogram (ECG), pulse oximetry, and non-invasive blood pressure monitoring (IntelliVue MP50; Philips Medizin Systeme Böblingen $\mathrm{GmbH}$, Böblingen, Germany). Additionally, the R-R intervals and plethysmographic blood pressure measurement were continuously registered (Task Force Monitor; CNSystems Medizintechnik AG, Graz, Austria).

The patients were prehydrated with $3 \mathrm{ml} / \mathrm{kg}$ of an isotonic crystalloid solution, followed by 2 to $3 \mathrm{ml} / \mathrm{kg}$ per hour. Anesthesia was induced with 10 to $20 \mu \mathrm{g}$ of remifantanil and etomidate until loss of consciousness, and tracheal intubation was facilitated by rocuronium $0.6 \mathrm{mg} / \mathrm{kg}$. Anesthesia was maintained by remifantanil $2.5 \mu \mathrm{g} / \mathrm{kg}$ per minute and propofol 0.05 to $0.06 \mathrm{mg} / \mathrm{kg}$ per minute as clinically required.

The patients were mechanically ventilated with a constant tidal volume of 7 to $8 \mathrm{ml} / \mathrm{kg}$, a positive end-expiratory pressure of 5 millibars, an inspiratory/expiratory ratio of $1: 1$, and a respiratory rate of 10 to 12 per minute to maintain an end-tidal $\mathrm{pCO}_{2}$ (partial pressure of carbon dioxide) of $35 \mathrm{~mm} \mathrm{Hg}$ at an $\mathrm{FiO}_{2}$ (fraction of inspired oxygen) of 0.5 throughout the entire study period.

A norepinephrine infusion was administered if required to maintain an SBP of $90 \mathrm{~mm} \mathrm{Hg}$.

Bipolar electrode catheters were placed in the right atrial appendage, the right ventricle, and via the coronary sinus in a posterior or lateral venous branch.

Three-minute recordings of ECG and arterial blood pressure were performed in a hemodynamic steady state during right ventricular and biventricular pacing. ECG and blood pressure were sampled at $1,000 \mathrm{~Hz}$ and stored on the hard disk of a personal computer.

Frequency-domain analysis of SBP and PP variability was performed in accordance with the suggestions of the Task Force of the European Society of Cardiology and the North American Society of Pacing and Electrophysiology [8]. Signals were inspected visually and checked for artifacts and heterotopic beats that would have been removed by interpolation by means of interactive software. Time series were computed with SBP and PP. Stationarity of each period was checked by the reverse arrangement test described by Bendat and Piersol [9]. Data were resampled at $4 \mathrm{~Hz}$ using a moving 500-millisecond-wide rectangular window. After substraction of the mean value of the sample data, removal of residual linear trends, and application of a cosine function (Hanning window) to avoid distortions of the estimated spectra [9], discrete Fourier analysis was performed for three 50\% overlapping windows, and the results were subsequently averaged. The area under the curve was calculated for the frequency component of respiration (respiratory frequency $\pm 0.025 \mathrm{~Hz}$ ).

Additionally, time-domain analysis of PP was performed. The three-minute data files were divided into 7.5-second periods. The difference between the minimum and maximum values of $\mathrm{PP}$, normalized by the mean of the two values and expressed as a percentage, was calculated in each window. The results of the 24 periods were subsequently averaged.

Statistical analysis was performed using commercially available software (SPSS for Windows, version 12.0; SPSS Inc., Chicago, IL, USA). The data were checked for normal distribution by means of the Lilliefors modification of the KolmogorovSmirnov test. The results of spectral power analysis were normally distributed after logarithmic transformation. Data are presented as mean \pm standard deviation or as median (25th percentile; 75th percentile). Data were compared using the Student $t$ test for paired data or the Wilcoxon signed rank test, as appropriate. An $\alpha$ error of 0.05 was considered significant.

\section{Results}

Demographic data and the characteristics of the patients are presented in Table 1. Five patients required norepinephrine up to a dosage of $2 \mu \mathrm{g} /$ minute intraoperatively to maintain an SBP of $90 \mathrm{~mm} \mathrm{Hg}$. The results of the hemodynamic measurements are presented in Table 2. Because the frequency of pacing did not change between right ventricular and biventricular pacing, the mean R-R interval was identical at the two sample points. Systolic, mean, and diastolic blood pressures did not change significantly between right ventricular and biventricular pacing. $\mathrm{PP}$ increased by $2 \mathrm{~mm} \mathrm{Hg}$ during biventricular pacing. This increase, however, did not reach statistical significance ( $p=$ 0.08). 
Table 1

\begin{tabular}{lc}
\hline Demographic data & $69 \pm 6$ \\
\hline Age (years) & $172 \pm 9$ \\
\hline Height $(\mathrm{cm})$ & $81 \pm 15$ \\
Weight $(\mathrm{kg})$ & 8 \\
Dilated cardiomyopathy $(n)$ & 11 \\
Ischemic heart disease $(n)$ & $24 \pm 6$ \\
LVEF (percentage) & 17 \\
Beta-receptor blockers $(n)$ & 18 \\
ACE inhibitors/Angiotensin II blockers $(n)$ & 7 \\
Amiodarone $(n)$ & 19 \\
Diuretics $(n)$ & 7 \\
Cardiac glycosides $(n)$ & 19
\end{tabular}

Data are reported as either mean \pm standard deviation or as frequency distributions $(n)$. ACE, angiotensin-converting enzyme; LVEF, left ventricular ejection fraction.

Exemplary registrations of $\mathrm{PP}$, recorded during right ventricular and biventricular pacing, and their related power spectra are demonstrated in Figure 1. The results of the frequencydomain and time-domain analyses are reported in Table 2 . The area under the curve in the respiratory frequency component of SBP and PP increased significantly $(p<0.01)$ during biventricular pacing, with a 1.5-fold increase in the geometric mean of SBP variability (right ventricular pacing, $0.563 \mathrm{~mm} \mathrm{Hg}^{2}$; biventricular pacing, $0.844 \mathrm{~mm} \mathrm{Hg}^{2}$ ) and a 1.8-fold increase in the geometric mean of PP variability (right ventricular pacing, $0.281 \mathrm{~mm} \mathrm{Hg}$; biventricular pacing, $0.516 \mathrm{~mm} \mathrm{Hg}^{2}$ ). The analysis of PP variation in the time domain, determined as the difference between the highest and lowest values and normalized by the mean value, revealed a 1.3-fold increase from 5.3\% to $6.9 \%$ during biventricular pacing $(p<0.01)$.

\section{Discussion}

Respiratory fluctuations in PP during mechanical ventilation are an expression of respiratory changes in left ventricular stroke volume: due to a decrease in right ventricular preload and an increase in right ventricular afterload, right ventricular stroke volume decreases during inspiration. Left ventricular stroke volume decreases with a delay of one to two heartbeats and is additionally modified by a variety of factors, such as a decrease in left ventricular afterload during inspiration [10-12].

Most studies dealing with PP variation have focused on the influence of the volume status on respiratory fluctuations: an increase in preload is related to a rightward shift of the cardiac operating point on the Frank-Starling curve with the consequence that a patient who is operating on the steep portion of the curve may operate on the flat portion. This change in the position on the Frank-Starling curve is related to a decrease in

Table 2

Hemodynamic variables during right ventricular and biventricular pacing

\begin{tabular}{|c|c|c|c|}
\hline & Right ventricular pacing & Biventricular pacing & $P$ value \\
\hline R-R interval (milliseconds) & $864 \pm 94$ & $864 \pm 93$ & 0.94 \\
\hline Systolic blood pressure $(\mathrm{mm} \mathrm{Hg})$ & $98 \pm 18$ & $100 \pm 18$ & 0.35 \\
\hline Mean blood pressure $(\mathrm{mm} \mathrm{Hg})$ & $75 \pm 13$ & $75 \pm 14$ & 0.75 \\
\hline Diastolic blood pressure $(\mathrm{mm} \mathrm{Hg})$ & $65 \pm 13$ & $64 \pm 13$ & 0.88 \\
\hline Pulse pressure $(\mathrm{mm} \mathrm{Hg})$ & $33 \pm 10$ & $35 \pm 11$ & 0.08 \\
\hline Respiratory systolic blood pressure variability $\left(\mathrm{ln} \mathrm{mm} \mathrm{Hg}^{2}\right)$ & $-0.57 \pm 1.42$ & $-0.17 \pm 1.37$ & 0.002 \\
\hline Respiratory pulse pressure variability $\left(\mathrm{In} \mathrm{mm} \mathrm{Hg}^{2}\right)$ & $-1.27 \pm 1.74$ & $-0.66 \pm 1.48$ & 0.002 \\
\hline Pulse pressure variation (percentage) & $5.3(3.1 ; 12.3)$ & $6.9(4.7 ; 16.4)$ & 0.008 \\
\hline
\end{tabular}

Data are reported as mean \pm standard deviation or as median (25th percentile; 75th percentile). Results of power spectral analysis are logarithmically transformed. 


\section{RV Pacing}


BV Pacing
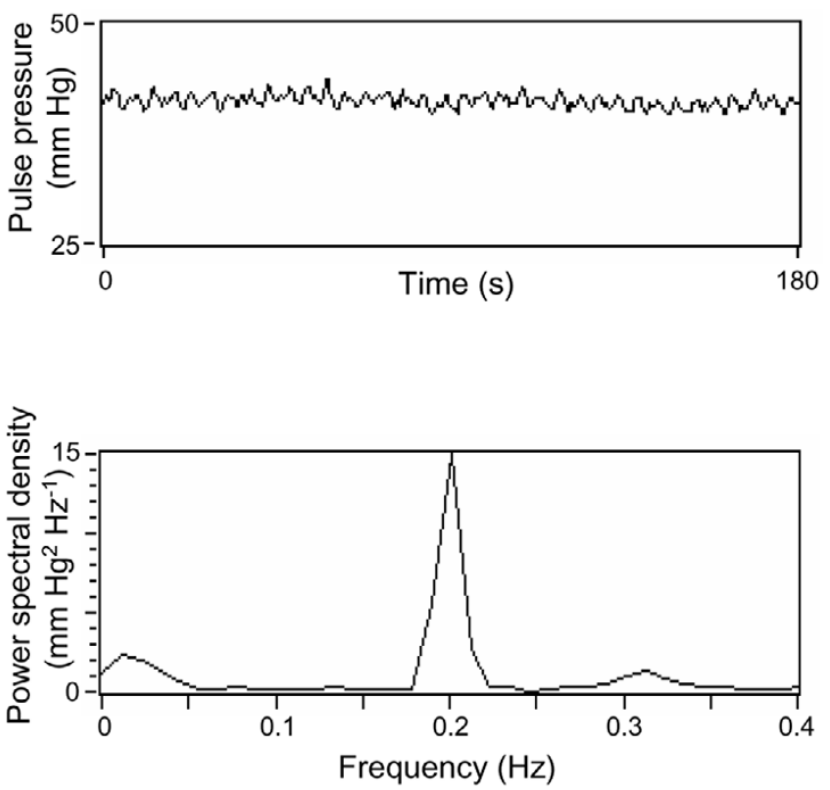

Exemplary three-minute registrations of pulse pressure (PP), recorded during right ventricular and biventricular pacing, and their related power spectra. The patient was ventilated at a frequency of $0.2 \mathrm{~Hz}$. In this patient, mean PP and the respiratory fluctuations of PP increased markedly during biventricular pacing. BV, biventricular; RV, right ventricular.

the respiratory fluctuation of PP.

In contrast to previous studies, we did not focus on the influence of the intravascular volume on the position of the cardiac operating point on the Frank-Starling curve, but on the influence of cardiac performance on the slope of the preload/ stroke volume relationship. A decrease in ventricular contractility decreases the slope of the relationship between enddiastolic volume and stroke volume [13]. Thus, the respiratory fluctuations of stroke volume and PP should decrease in the failing heart in mechanically ventilated patients. Conversely, an improvement in cardiac performance should create an increase in the respiratory fluctuations of PP: a patient with heart failure who operates on a flattened Frank-Starling curve may operate on a much steeper portion of the new curve.

Our results confirm this physiological model. The respiratory fluctuations of PP increased significantly during biventricular pacing in our patients with severely impaired myocardial function.

\section{The influence of biventricular pacing on the dynamic behavior of PP}

In previous studies, PP variation derived by time-domain analysis was markedly decreased when compared to patients with normal or moderately impaired myocardial function $[3,14]$. Reuter and colleagues [1] compared stroke volume variation of patients with normal left ventricular function with that of patients with impaired left ventricular function and found that stroke volume variation was decreased in patients with an ejection fraction of less than $35 \%$, but without statistical significance. Both patient groups with normal myocardial function and with impaired myocardial function showed significant fluid responsiveness (that is, a decrease in PP variation after fluid loading) [1]. In our patients with severely impaired cardiac function, PP variation remained (even during biventricular pacing) below the threshold value indicating fluid responsiveness, which was determined to be 10\% [14]. Because our patients were suffering from advanced drug-refractory heart failure, we did not test the effect of fluid loading on PP variability.

In addition to time-domain analysis, we assessed blood pressure variability by spectral analysis and were thus able to analyze the influence of ventilation on blood pressure at the specific frequency of respiration. Respiratory fluctuations in SBP are less closely related to changes in stroke volume than are respiratory fluctuations in PP $[10,12]$. Nevertheless, the respiratory fluctuations of SBP behaved comparably to those of PP in our patients.

Previously, in adults anesthetized with propofol, we observed that the respiratory fluctuations of blood pressure were superimposed by major fluctuations, which were located at a significantly lower frequency component than the mechanical ventilation or the Mayer waves [15]. In these patients, the difference between maximum and minimum SBPs (taken as a 
measure of volume responsiveness) could have provided a misleading result not related to the respiratory changes in arterial pressure if the time interval had been much longer than the respiratory cycle length. Our results do not confirm this concern: we determined SBP and PP variation at a time interval of 7.5 seconds (in accordance with previous studies [3]) and observed a similar behavior of fluctuations of SBP and PP calculated in the time domain and in the frequency domain.

The interaction of respiratory changes in stroke volume and intrathoracic and transpulmonary pressures is of major importance when interpreting respiratory fluctuations of blood pressure [16]. A previous study indicated that an increase in tidal volume was associated with an increase in PP variation [14]. Other investigators found that the stroke volume variation, assessed during ventilation with a large tidal volume $(15 \mathrm{ml} /$ $\mathrm{kg}$ ), was a good predictor of volume responsiveness [17], whereas our group had inconsistent results when ventilating patients with smaller tidal volumes $[3,18]$. In the present study, we ventilated patients with a tidal volume of 7 to $8 \mathrm{ml} / \mathrm{kg}$ body weight at a respiration rate of 10 to 12 per minute. Larger tidal volumes would have required a decrease in respiration rate in order to maintain normoventilation. It is well known that in the awake, spontaneously breathing human the respiratory hemodynamic fluctuations increase at lower breathing frequencies (despite constant tidal volume) and have a maximum of approximately $0.1 \mathrm{~Hz}$ (that is, the frequency of the spontaneously occurring Mayer waves) [19-21]. Until now, whether this phenomenon also occurs under general anesthesia has not been investigated. In a previous study, we found that the hemodynamic fluctuations of approximately $0.1 \mathrm{~Hz}$ were markedly depressed under propofol anesthesia [15]. However, it remains to be examined whether not only the tidal volume, but also the respiratory frequency, is relevant when analyzing respiratory hemodynamic fluctuations during anesthesia in mechanically ventilated patients.

\section{The influence of biventricular pacing on static changes in PP}

Several studies reported an improvement in $\mathrm{dP} / \mathrm{dt}_{\max }$ as a measure of isovolumic systolic function and in PP as a surrogate of stroke volume during biventricular pacing in patients with heart failure and intraventricular conduction delay $[5,22]$. Consequently, these parameters are frequently used to assess the optimal position of the left ventricular electrode and the stimulation configuration [23]. The PP of our patients during right ventricular pacing is comparable to that reported by other authors during baseline conditions [5]. However, we found a modest increase in mean PP during biventricular pacing of approximately $2 \mathrm{~mm} \mathrm{Hg}$, which was not significant. Other authors have reported an increase of 4 to $8 \mathrm{~mm} \mathrm{Hg}[6,22]$. It is not clear whether these differences are related to the electrode positioning, regional differences in ischemic lesions, or methodological differences in blood pressure recording. Investigators who determined cardiac output invasively or by echocardiography found a median increase of $8 \%$ and mean increases of $10 \%$ and $15 \%$, respectively, during biventricular pacing $[7,24,25]$. Because our patients met the characteristics of patients who typically show an improvement in cardiac performance during biventricular pacing, we avoided the potential risk that is related to the invasive measurements of cardiac output.

\section{Limitations of the study}

The absence of invasive measurement of cardiac output for the above-mentioned reason may be a limitation of the study. We measured beat-to-beat blood pressure by a finger plethysmographic method, which is calibrated by the oscillometric measurement of blood pressure (Task Force Monitor; CNSystems). This methodology showed satisfactory precision in the assessment of blood pressure changes compared to intraarterial measurement [26,27]. Pinna and colleagues [28] observed sufficient agreement in the frequency band of respiration in patients with chronic heart failure when comparing the power spectra of blood pressure recorded invasively and by the finger plethysmographic method, respectively. Nevertheless, we cannot rule out that results assessed by finger plethysmographic measurements are not fully comparable to those of studies using invasive blood pressure measurement.

\section{Conclusion}

Our findings indicate that changes in cardiac performance have a significant influence on respiratory fluctuations in PP. This interaction should be considered when interpreting PP variation in the clinical setting. Our results suggest, furthermore, that respiratory PP variability is a parameter that is much more sensitive to an improvement in systolic cardiac performance than changes in the mean value of PP in mechanically ventilated patients with heart failure associated with an intraventricular conduction disorder. Whether this parameter might be helpful in guiding the positioning of electrodes and optimizing the configuration of biventricular stimulation in mechanically ventilated patients remains to be determined.

\section{Key messages}

- Cardiac performance has a considerable impact on the respiratory fluctuations of pulse pressure in mechanically ventilated patients with heart failure.

- Cardiac resynchronization therapy is associated with minor changes in blood pressure but with major changes in respiratory fluctuations of pulse pressure in mechanically ventilated patients.

\section{Competing interests}

The authors declare that they have no competing interests.

\section{Authors' contributions}

CK designed the study, processed and analyzed the data, and wrote the manuscript. JS contributed to the study design and 
interpretation of data. SL collected the clinical data and revised the manuscript critically. KS contributed to the acquisition of data. JS-R collected the clinical data and contributed to data interpretation. CW was involved in data interpretation and drafting the manuscript. All authors read and approved the final manuscript.

\section{Acknowledgements}

This study was supported by Guidant $\mathrm{GmbH}$ (financial support for purchase of Task Force Monitor, CNSystems Medizintechnik AG).

\section{References}

1. Reuter DA, Kirchner A, Felbinger TW, Weis FC, Kilger E, Lamm P, Goetz AE: Usefulness of left ventricular stroke volume variation to assess fluid responsiveness in patients with reduced cardiac function. Crit Care Med 2003, 31:1399-1404.

2. Kramer A, Zygun D, Hawes H, Easton P, Ferland A: Pulse pressure variation predicts fluid responsiveness following coronary artery bypass surgery. Chest 2004, 126:1563-1568.

3. Wiesenack C, Fiegl C, Keyser A, Prasser C, Keyl C: Assessment of fluid responsiveness in mechanically ventilated cardiac surgical patients. Eur J Anaesthesio/ 2005, 22:658-665.

4. Pinsky MR: Cardiopulmonary monitoring. Curr Opin Crit Care 2006, 12:218.

5. Auricchio A, Stellbrink C, Block M, Sack S, Vogt J, Bakker P, Klein $\mathrm{H}$, Kramer A, Ding J, Salo R, et al.: Effect of pacing chamber and atrioventricular delay on acute systolic function of paced patients with congestive heart failure. The Pacing Therapies for Congestive Heart Failure Study Group. The Guidant Congestive Heart Failure Research Group. Circulation 1999, 99:2993-3001.

6. Nelson GS, Berger RD, Fetics BJ, Talbot M, Spinelli JC, Hare JM, Kass DA: Left ventricular or biventricular pacing improves cardiac function at diminished energy cost in patients with dilated cardiomyopathy and left bundle-branch block. Circulation 2000, 102:3053-3059.

7. Boriani G, Saporito D, Biffi M, Martignani C, Valzania C, Diemberger I, Ziacchi M, Artale P, Grigioni F, Bacchi L, et al:: Acute and chronic haemodynamic effects of biventricular pacing and of switching to different pacing modalities in heart failure patients. Int J Cardiol 2005, 110:318-323.

8. Task Force of the European Society of Cardiology and the North American Society of Pacing and Electrophysiology: Heart rate variability. Standards of measurement, physiological interpretation, and clinical use. Circulation 1996, 93:1043-1065.

9. Bendat JS, Piersol AG: Random Data: Analysis and Measurement Procedures 2nd edition. New York: Wiley; 1986

10. Innes JA, De Cort SC, Kox W, Gut A: Within-breath modulation of left ventricular function during normal breathing and positive-pressure ventilation in man. J Physiol 1993, 460:487-502.

11. Vieillard-Baron A, Chergui K, Augarde R, Prin S, Page B, Beauchet $\mathrm{A}$, Jardin $\mathrm{F}$ : Cyclic changes in arterial pulse during respiratory support revisited by Doppler echocardiography. Am J Respir Crit Care Med 2003, 168:671-676.

12. Michard $\mathrm{F}$ : Changes in arterial pressure during mechanical ventilation. Anesthesiology 2005, 103:419-428.

13. Michard F, Teboul JL: Predicting fluid responsiveness in ICU patients: a critical analysis of the evidence. Chest 2002, 121:2000-2008

14. Charron C, Fessenmeyer C, Cosson C, Mazoit JX, Hebert JL, Benhamou D, Edouard AR: The influence of tidal volume on the dynamic variables of fluid responsiveness in critically ill patients. Anesth Analg 2006, 102:1511-1517.

15. Keyl C, Schneider A, Dambacher M, Wegenhorst U, Ingenlath $M$, Gruber M, Bernardi L: Dynamic cardiocirculatory control during propofol anesthesia in mechanically ventilated patients. Anesth Analg 2000, 91:1188-1195.

16. Pinsky MR: Probing the limits of arterial pulse contour analysis to predict preload responsiveness. Anesth Analg 2003, 96:1245-1247.

17. Reuter DA, Felbinger TW, Schmidt C, Kilger E, Goedje O, Lamm $P$, Goetz AE: Stroke volume variations for assessment of car- diac responsiveness to volume loading in mechanically ventilated patients after cardiac surgery. Intensive Care Med 2002 28:392-398

18. Wiesenack C, Prasser C, Rodig G, Keyl C: Stroke volume variation as an indicator of fluid responsiveness using pulse contour analysis in mechanically ventilated patients. Anesth Analg 2003, 96:1254-1257.

19. Saul JP, Berger RD, Albrecht $P$, Stein SP, Chen MH, Cohen RJ: Transfer function analysis of the circulation: unique insights into cardiovascular regulation. Am J Physiol 1991, 261: $\mathrm{H} 1231-\mathrm{H} 1245$

20. Novak V, Novak P, de Champlain J, Le Blanc AR, Martin R, Nadeau $\mathrm{R}$ : Influence of respiration on heart rate and blood pressure fluctuations. J Appl Physiol 1993, 74:617-626.

21. Pagani M, Montano N, Porta A, Malliani A, Abboud FM, Birkett C Somers VK: Relationship between spectral components of cardiovascular variabilities and direct measures of muscle sympathetic nerve activity in humans. Circulation 1997, 95:1441-1448.

22. Nelson GS, Curry CW, Wyman BT, Kramer A, Declerck J, Talbot M, Douglas MR, Berger RD, McVeigh ER, Kass DA: Predictors of systolic augmentation from left ventricular preexcitation in patients with dilated cardiomyopathy and intraventricular conduction delay. Circulation 2000, 101:2703-2709.

23. Butter C, Auricchio A, Stellbrink C, Fleck E, Ding J, Yu Y, Huvelle $E$, Spinelli J: Effect of resynchronization therapy stimulation site on the systolic function of heart failure patients. Circulation 2001, 104:3026-3029.

24. Foster $A H$, Gold MR, McLaughlin JS: Acute hemodynamic effects of atrio-biventricular pacing in humans. Ann Thorac Surg 1995, 59:294-300.

25. Sundell J, Engblom E, Koistinen J, Ylitalo A, Naum A, Stolen KO Kalliokoski R, Nekolla SG, Airaksinen KE, Bax JJ, et al:: The effects of cardiac resynchronization therapy on left ventricular function, myocardial energetics, and metabolic reserve in patients with dilated cardiomyopathy and heart failure. J Am Coll Cardiol 2004, 43:1027-1033.

26. Parati G, Cassadei R, Gropelli A, Di Rienzo M, Mancia G: Comparison of finger and intra-arterial blood pressure monitoring in rest and during laboratory tests. Hypertension 1989, 13:647-655

27. Petersen ME, Williams TR, Sutton R: A comparison of non-invasive continuous finger blood pressure measurement (Finapres) with intra-arterial pressure during prolonged head-up tilt. Eur Heart J 1995, 16:1641-1654.

28. Pinna GD, La Rovere MT, Maestri R, Mortara A, Bigger JT, Schwartz PJ: Comparison between invasive and non-invasive measurements of baroreflex sensitivity. Eur Heart $J$ 2000, 21:1522-1529. 